\title{
Effect of Solutol HS 15 in Solid Dispersions of Pioglitazone Hydrochloride: in vitro and in vivo Evaluation
}

\author{
R. P. SWAIN ${ }^{1 *}$, B. B. SUBUDHI ${ }^{1}$ AND P. RAMESH ${ }^{1}$
}

Department of Pharmaceutical Technology, Maharajah's College of Pharmacy, Vizianagaram-535 002, ${ }^{1}$ Drug Development and Analysis Laboratory, School of Pharmaceutical Sciences, Siksha O Anusandhan University, Bhubaneswar-751 003, India

\author{
Swain et al.: Effect of Solutol HS 15 on in vitro/in vivo Study
}

\begin{abstract}
Poor aqueous solubility and slow dissolution rate adversely affects bioavailability of pioglitazone hydrochloride. This study was undertaken to improve dissolution and bioavailability of pioglitazone hydrochloride through preparation of stable solid dispersions by melting and solvent evaporation method using Solutol HS 15. Solubility of pioglitazone hydrochloride was enhanced by 9 to 49 fold as the drugcarrier ratio was increased in solid dispersions as compared to pure drug. Furthermore, compared to pure drug or physical mixture, solid dispersions significantly improved the dissolution rate and the extent of drug release. Solid dispersions at 1:7 drug:Solutol HS 15 weight ratio showed complete and rapid drug release within 15 min at pH 1.2. The Fourier-transform infrared spectrum revealed the chemical compatibility with Solutol HS 15. Differential scanning calorimetry and X-ray powder diffraction pattern revealed a change in crystallinity to amorphous state that supported the enhancement of solubility of pioglitazone hydrochloride with Solutol HS 15. In vivo test showed that Solutol HS 15-based solid dispersions showed higher $\mathrm{AUC}_{0-\mathrm{t}}$ and $\mathrm{C}_{\max }$, which was $\sim 4$ times higher than that of pure pioglitazone $(\mathrm{p}<0.05)$ implying solid dispersions could be effective in increasing the bioavailability. In conclusion, solid dispersions with Solutol HS 15 prepared using solvent evaporation method appeared to be a promising technique to improve the dissolution, bioavailability and stability of pioglitazone hydrochloride.
\end{abstract}

Key words: Pioglitazone HCI, Solutol HS 15, pharmacokinetic, recrystallization, solid dispersion

Oral route is the most preferred route of administration for majority of the drugs ${ }^{[1]}$. Unfortunately many drug candidates suffer from poor pharmacokinetics and bioavailability, following oral delivery due to poor solubility ${ }^{[2]}$. Biopharmaceutics classification system (BCS) class II drugs often exhibit dissolution ratelimited absorption leading to low and variable oral bioavailability. There is a need to enhance the solubility without compromising for stability and activity of the drugs.

Pioglitazone hydrochloride ( $\mathrm{HCl})$, a BCS class II drug is used as a potent and highly selective agonist for peroxisome proliferator-activated receptor gamma in type II diabetes ${ }^{[3-5]}$. The poor aqueous solubility and dissolution rate of pioglitazone $\mathrm{HCl}$ is a major hurdle in its bioavailability and therapeutic application ${ }^{[6,7]}$. To enhance its solubility, approaches including inclusion complexation $^{[8-10]}$, micellar solubilization ${ }^{[11]}$, co-solvent solubilisation $^{[12]}$, solid dispersion ${ }^{[13-15]}(\mathrm{SD})$ and spray

*Address for correspondence E-mail: ranjit.prasad797@gmail.com

March-April 2019 drying ${ }^{[16]}$ have been evaluated. High thermodynamic stability of the first and second generation polymeric carriers lowers the dissolution rate, recrystallization and precipitation, which were the limitations of the SDs. Amorphous SDs is a promising strategy to increase the bioavailability of poorly soluble drugs by improving their rate and extent of dissolution ${ }^{[17]}$. However the earlier reported SDs of pioglitazone had stability issues due to precipitation and recrystallization, which negatively impacted solubility leading to inadequate dissolution. Besides, there are not enough reports to correlate the in vitro dissolution to in vivo bioavailability. Hence, there is an unmet need to

This is an open access article distributed under the terms of the Creative Commons Attribution-NonCommercial-ShareAlike 3.0 License, which allows others to remix, tweak, and build upon the work non-commercially, as long as the author is credited and the new creations are licensed under the identical terms

Accepted 15 February 2019

Revised 22 September 2018

Received 24 March 2018

Indian J Pharm Sci 2019;81(2):317-325 
properly adopt the SDs to improve stability, solubility and bioavailability of pioglitazone $\mathrm{HCl}$. Amorphous SDs with classical surfactant carriers have attracted attention of many researchers. However, in many instances, the amorphous form is thermodynamically unstable and tends to crystallize during storage leading to decreased solubility ${ }^{[18]}$. Thus careful choice of carriers and methods of preparation of SDs are critical for enhancing the solubility of pioglitazone $\mathrm{HCl}$ while overcoming the limitations of previous approaches. Solutol HS 15 is a third generation surfactant carrier used to improve the solubility and bioavailability ${ }^{[18,19]}$. Thus its use was optimised to achieve the desired attributes. Surface active carriers (non-polymeric) like Cremophor RH 40, Poloxamers were proposed to influence the rate and extent of drug absorption by altering the thermodynamic activity, solubility, diffusion, disintegration and dissolution rate of a drug. Furthermore, surface active carriers can exert direct effects on biological membranes leading to modulation of drug transport across the membrane ${ }^{[20]}$. Melting (MM) and solvent evaporation (SM) methods are the most scalable industrial technologies for manufacture of SDs. Homogeneous mixing of the drug and carrier in molten state without any solvent is the advantage of $\mathrm{MM}^{[21,22]}$. Prevention of decomposition of the drug and carrier at high temperature and molecular mixing in solution stage are the advantages of $\mathrm{SM}^{[21,22]}$. The influence of carriers on crystallization behaviour, impact on in vitro dissolution and in vivo performance has not been investigated to the best of our knowledge. Therefore, this study was aimed to evaluate the prepared SDs using a surfactant carrier and pioglitazone $\mathrm{HCl}$. The prepared formulations were thoroughly evaluated to understand the impact on in vitro and in vivo performance to justify their application in oral drug delivery.

\section{MATERIALS AND METHODS}

Pioglitazone $\mathrm{HCl}$ was obtained as a gift sample from Aurobindo Pharma Ltd. (Hyderabad, India). Cremophor RH40 (polyethoxylated castor oil), Solutol HS 15 (macrogol 15 hydroxystearate), Poloxamer 188, Poloxamer 407, hydroxypropyl methylcellulose (HPMC), polyethylene glycol (PEG) 4000, polyvinylpyrrolidone (PVP) K-15 were purchased from Yarrow Chem Products, Mumbai, India. Ammonium acetate was purchased from Merck Specialities Pvt., Ltd. (Mumbai, India). Acetonitrile was purchased from the Thermo Fisher Scientific India Pvt., Ltd. (Mumbai,
India). All other chemicals and reagents were purchased from Lotus Enterprises, Andhra Pradesh, India.

\section{SDs preparation:}

Initially SDs were prepared using SM with HPMC, PEG 4000, PVP K-15, Cremophor RH 40, Solutol HS 15, Poloxamer 188 and 407 carriers in 1:5 weight ratio (drug:polymer). The aqueous solubility was determined for screening of carrier. After screening, SDs of pioglitazone $\mathrm{HCl}$ were formulated with Solutol HS 15 in various drug/polymer weight ratios (1:1, 1:3, 1:5, 1:7 and 1:9) by physical mixing (PM), MM and SM. Pioglitazone $\mathrm{HCl}$ and Solutol HS 15 were mixed in a glass mortar for 10 min to obtain PMs. In MM, carrier was heated at $10^{\circ}$ above the melting point of the carrier. Drug was dispersed in the melted carrier with continuous agitation. The blend mass was immediately transferred in crushed ice bath, so that the crystalline drug will convert amorphous. SDs were also developed by SM. Ethanol $(10 \mathrm{ml})$ was used to solubilise the pioglitazone $\mathrm{HCl}$ and carrier individually by continuous stirring with a glass rod at room temperature and mixed together. Solvent was removed under reduced pressure using a rotary evaporator (model R-215, Buchi, Switzerland) and kept at $40^{\circ}$ in hot air oven (KOMS3, KEMI, India) for complete evaporation of ethanol. The products were scraped, pulverized gently with a mortar and pestle and sieved through 40\# mesh sieve. The samples were stored in screw cap glass vials and kept in a desiccator until further uses ${ }^{[17,21]}$.

\section{Solubility test:}

Sample amount equivalent to $10 \mathrm{mg}$ of pioglitazone $\mathrm{HCl}$ was added in $10 \mathrm{ml}$ of distilled water. The samples were sealed in triplicate and shaken for $48 \mathrm{~h}$ at $37 \pm 0.5^{\circ}$ in a thermostatically controlled horizontal shaker (model: RSB12, Remi Elektrotechnik Ltd., India). The resulting samples were filtered through a Millipore filter (pore size $0.45 \mu \mathrm{m}$ ), suitably diluted and assayed by following a valid reversed-phase high pressure liquid chromatography (HPLC, Agilent 1220 infinity series, software: Ezichrome elite, Agilent Technologies, USA) method $^{[23]}$.

\section{In vitro dissolution study:}

In vitro dissolution studies were performed in triplicate using the USP II dissolution test apparatus (Electrolab, Mumbai, Model no. TDT-08L) maintained at temperature of $37 \pm 0.5^{\circ}$ at $75 \mathrm{rpm}$ speed in $900 \mathrm{ml}$ of $0.1 \mathrm{~N} \mathrm{HCl}$. Pure drug and SD containing an equivalent to $15 \mathrm{mg}$ of the pioglitazone $\mathrm{HCl}$ were introduced 
into dissolution medium. At the predetermined time $(5,10,15,30,45,60 \mathrm{~min})$ intervals, $5 \mathrm{ml}$ samples were withdrawn, filtered through membrane filter ( $0.45 \mu \mathrm{m}$, Millipore). Immediately $5 \mathrm{ml}$ of fresh medium maintained at $37 \pm 0.5^{\circ}$ was replaced to maintain its constant volume. The samples were suitably diluted wherever necessary and analysed for pioglitazone $\mathrm{HCl}$ using HPLC method.

\section{Fourier-transform infrared spectroscopy (FTIR):}

FTIR spectra can be used to detect drug-polymer interactions by following the shift in vibrational or stretching bands of key functional groups. FTIR spectra were obtained by using diamond ATR spectrophotometer (model: Cary 630, Agilent technologies, Germany). All the samples (2-5 mg) were placed in diamond ATR sample holder and scanned over a wave number range of $500-4000 \mathrm{~cm}^{-1}$.

\section{Differential scanning calorimetry (DSC):}

DSC was performed for the pure drug pioglitazone, pure polymers, PMs and SDs using a DSC apparatus (Pyris Diamond thermo gravimetry/differential thermal analysis, PerkinElmer, Singapore). The powdered sample (3-5 mg) was hermetically sealed in aluminium pans and lids were crimped using a TA crimper and heated at a constant rate of $10 \% \mathrm{~min}$ over a temperature range of $30-250^{\circ}$, using nitrogen as purge gas at a flow rate of $150 \mathrm{ml} / \mathrm{min}$. Platinum crucible was used with alpha alumina powder as reference.

\section{X-ray diffraction (XRD):}

XRD of pure drug, carrier and formulations were performed at room temperature with an X-ray diffractometer (Ultima-III, Rigaku, Japan). Monochromatic $\mathrm{Cu} \mathrm{K \alpha}$ radiation $(\lambda=1.5418 \AA$ ) $)$ was obtained with a Ni-filtration and a system of diverging and receiving slides of $0.5^{\circ}$ and $0.1 \mathrm{~mm}$, respectively. The diffraction pattern was measured with a voltage of $40 \mathrm{kV}$ and current of $30 \mathrm{~mA}$ over a $2 \theta$ range of $0-55^{\circ}$ using a step size of $0.02^{\circ}$ at a scan speed of $1 \mathrm{~s} / \mathrm{step}$.

\section{Stability test:}

Stability testing $\left(25^{\circ}\right.$ and $\left.60 \% \mathrm{RH}\right)$ of SDs (SM) were performed as per the ICH guidelines in the stability chamber (Remi Instruments, India) ${ }^{[24]}$. Samples were analysed periodically for $3 \mathrm{mo}$ and subjected to solubility and XRD analysis to explore the changes in the system.

\section{Quantification/determination of pioglitazone $\mathrm{HCl}$ :}

A valid reversed-phase HPLC method was followed for quantification of pioglitazone $\mathrm{HCl}$ for in vitro studies ${ }^{[25]}$. For in vivo analysis the same method was modified and verified before quantification of pioglitazone $\mathrm{HCl}$ from plasma. A reversed-phase Agilent-TC- C18, $(250 \times 4.6 ; 5 \mu)$ column was used for separation. The mobile phase was $0.1 \mathrm{M}$ ammonium acetate $(\mathrm{pH} 4.0$, adjusted with acetic acid):acetonitrile $(70: 30, \mathrm{v} / \mathrm{v})$. The effluent was observed at $254 \mathrm{~nm}$ after a retention time of $3 \mathrm{~min}$ with a flow rate of $1.0 \mathrm{ml} / \mathrm{min}$. The assay was linear for pioglitazone $\mathrm{HCl}$ in the concentration range of 0.5 $80 \mu \mathrm{g} / \mathrm{ml}$ and the limit of detection was $0.05 \mu \mathrm{g}$.

\section{In vivo studies:}

The procedure for the in vivo study was approved by the Institutional Animal Ethical Committee (approval no: 02/IAEC/CIPS/2016-17) and appropriate guidelines for the use of animals were followed during the study. The objective of this study was to characterize the bioavailability of pioglitazone $\mathrm{HCl}$ from optimized SD as compared with the pure drug. Six male New Zealand rabbits (body weight $1.83 \pm 0.08 \mathrm{~kg}$ ) divided randomly into two groups $(\mathrm{n}=3)$, were fasted for $18 \mathrm{~h}$ prior to the experiments and given free access to the water. Pure pioglitazone $\mathrm{HCl}(2.5 \mathrm{mg} / \mathrm{ml}$ suspended in $0.5 \%$ methyl-cellulose prepared in water for injection) and $\mathrm{SD}$ of pioglitazone $\mathrm{HCl}$ :Solutol HS 15 prepared by $\mathrm{SM}$ at a dose equivalent to $2.5 \mathrm{mg} / \mathrm{kg}$ of pioglitazone $\mathrm{HCl}$ were administered orally ${ }^{[26]}$. Blood samples $(1 \mathrm{ml})$ were collected from marginal ear vein at time points of $0.5,1,2,4,8,12$ and $24 \mathrm{~h}$ post dose and transferred to ethylenediaminetetraacetic acid (EDTA)-coated tubes to prevent coagulation. Then blood samples were centrifuged at $5000 \mathrm{rpm}$ for $10 \mathrm{~min}$ and the obtained plasma samples were stored at $-80^{\circ}$ until analysis.

\section{Preparation of sample:}

Frozen plasma samples were thawed at ambient temperature prior to extraction. In polypropylene tubes $0.1 \mathrm{ml}$ of sample and $3 \mathrm{ml}$ of tetra butyl methyl ether was added for extraction. Then, the mixture was vortexed for $15 \mathrm{~min}$ and centrifuged at $8000 \mathrm{rpm}$ for $5 \mathrm{~min}$. The supernate was transferred to new polypropylene tubes and dried at $70^{\circ}$ for $20 \mathrm{~min}$ in a turbo evaporator (TurboVap LV, Biotage, NC USA). The residues were reconstituted with mobile phase and injected $(10 \mu \mathrm{l})$ into HPLC for analysis by a validated reversed-phase HPLC method. 


\section{Pharmacokinetic analysis:}

The primary pharmacokinetic parameters were performed by non-compartmental analysis. Maximum plasma concentration $\left(\mathrm{C}_{\max }\right)$, time to reach the peak plasma concentration $\left(\mathrm{T}_{\max }\right)$ and area under the plasma concentration-time curve $\left(\mathrm{AUC}_{0-t}\right)$ were obtained from plasma data analysis using the software program PKSolver ${ }^{[27]}$.

\section{Statistical analysis:}

All the mean values were presented with their standard deviations $(m e a n \pm S D)$. Statistically significant difference was determined using $t$-test at 0.05 level of significance and $\mathrm{p}$ value $<0.05$ was considered statistically significant ${ }^{[28]}$.

\section{RESULTS AND DISCUSSION}

SDs of pioglitazone $\mathrm{HCl}$ was prepared by $\mathrm{SM}$ using non-polymeric (Poloxamers, Solutol HS 15, Cremophor RH 40) and polymeric carriers (HPMC, PEG 4000, Povidone K-15) at the drug-carrier ratio of 1:5 and then their effectiveness in improving the solubility of pioglitazone $\mathrm{HCl}$ was evaluated (Table 1). Solutol HS 15 was the most effective to increase the solubility of pioglitazone $\mathrm{HCl}$ by approximately 33 fold whereas Cremophor RH 40 increased approximately 27 folds. Suitability of Solutol HS 15 has earlier been demonstrated with its physiological compatibility and safety ${ }^{[29]}$. Entrapment of drugs in the loose and porous spherical micelles has been the major contributor to its high solubilisation capacity. In contrast to this, HPMC was least effective polymer for improving solubility of pioglitazone $\mathrm{HCl}$ (Table 1). Poloxamer 188 and 407 due to surface active nature of carrier and swelling effect in water ${ }^{[30]}$ showed $\sim 17$ fold increase in solubility of pioglitazone $\mathrm{HCl}$. Appreciable enhancement of solubility was also observed with PEG 4000 and povidone K-15. Nevertheless, Solutol HS 15 was selected for further study as hydrophilic carrier due to higher solubilisation capacity.

Crystallinity and aqueous solubility of drug is affected by the preparation techniques and drug-carrier ratio $^{[31]}$. Considering this, the effect of amount of Solutol HS 15 on the solubility of pioglitazone $\mathrm{HCl}$ is illustrated in fig. 1. The solubility of pioglitazone $\mathrm{HCl}$ significantly $(\mathrm{p}<0.05)$ increased in $\mathrm{PMs}$, SMs and MMs with increase in concentration of the carrier. Concentration of Solutol HS 15 used in this study was higher than its critical micelle concentration ${ }^{[32]}$.
TABLE 1: SOLUBILITY OF PIOGLITAZONE $\mathrm{HCl}$ FROM DIFFERENT SD FORMULATIONS

\begin{tabular}{lcc}
\hline Formulations $(\mathrm{w} / \mathrm{w})$ & Solubility $(\mathrm{mg} / \mathrm{l})$ \\
\hline Pioglitazone $\mathrm{HCl}$ & (untreated powder) & $8.22 \pm 2.63$ \\
& Solutol HS 15 & $276.81 \pm 3.5$ \\
& Cremophor RH 40 & $219.93 \pm 4.68$ \\
& Poloxamer 188 & $136.05 \pm 3.51$ \\
Pioglitazone HCl: & Poloxamer 407 & $149.26 \pm 4.53$ \\
& PEG 4000 & $86.44 \pm 4.43$ \\
& Povidone K- 15 & $104.31 \pm 3.21$ \\
& HPMC & $37.80 \pm 4.69$ \\
\hline
\end{tabular}

Mean \pm SD $(n=3)$, $1: 5$ weight ratio of pioglitazone $\mathrm{HCl}$ and carrier

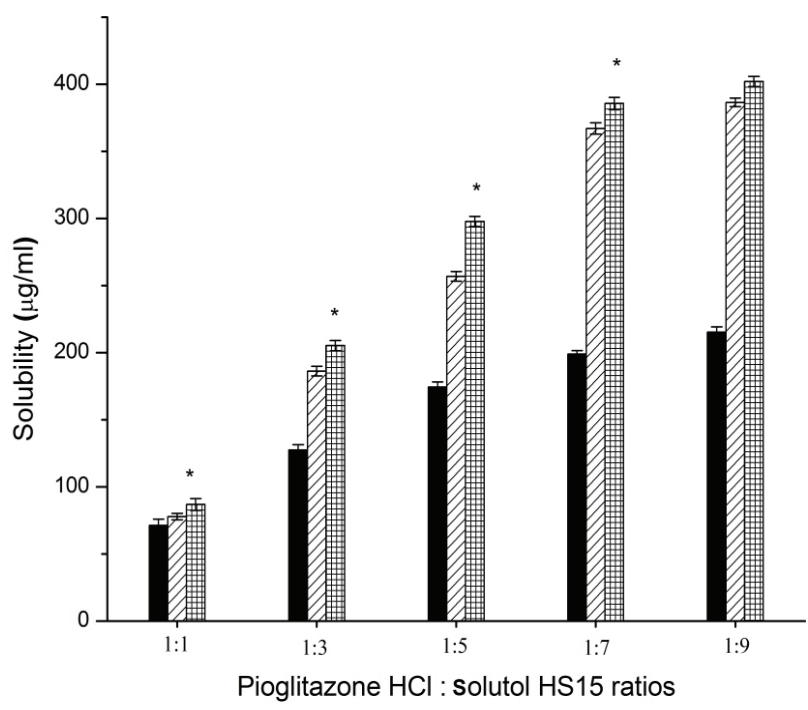

Fig. 1: Solubility of pioglitazone $\mathrm{HCl}$ from PM and SDs containing Solutol HS 15 in various proportions Solubility of pioglitazone $\mathrm{HCl}$ in physical mixture (PM) and solid dispersions (SDs) prepared by melting method (MM) and solvent evaporation method (SM) with Solutol HS 15 in various drug:carrier ratios. Mean $\pm \mathrm{SD}, \mathrm{n}=\mathbf{3},{ }^{*} \mathbf{p}<\mathbf{0 . 0 5}, \mathrm{PM}, \mathrm{W} / \mathrm{M}, \mathrm{MM}$, 無 SM

So, there was simultaneous increase in concentration of micelles along with increase in concentration of Solutol HS 15. SMs showed significant $(p<0.05)$ higher enhancement of solubility of pioglitazone $\mathrm{HCl}$ than its corresponding PMs. Formulations (1:3) showed approximately 2.40 fold more solubility enhancement than 1:1 formulations. However 1:5 formulations showed approximately 1.30 fold more pioglitazone $\mathrm{HCl}$ solubility (fig. 1). Approximately 1.80 fold solubility was decreased as the concentration of the Solutol increased twice. Pioglitazone $\mathrm{HCl}$ and Solutol HS 15 (1:7 weight ratio) showed significant enhancement $(\mathrm{p}<0.05)$ of solubility than lower ratios. In higher ratios (1:7, 1:9), SMs showed approximately twice higher solubility than PMs and almost similar in the lower ratios. MMs and SMs showed almost similar enhancement of solubility of pioglitazone $\mathrm{HCl}$ in all the chosen ratios. In comparison, solubility of pioglitazone 
$\mathrm{HCl}$ was increased up to $1: 7$ ratio but further there was no significant enhancement of solubility as the amount of Solutol was added (1:9) and further it become constant. Enhancement of solubility might be due to the conversion of crystalline to amorphous state. Thus, SD with 1:7 weight ratio was found to be optimum for further exploration.

This formulation was meant for the oral drug delivery. It comes across different $\mathrm{pH}$ conditions from oral cavity to intestine to systemic circulation. Thus, the progression of in vivo dissolution will be far more complicated than the results of in vitro dissolution studies with USP dissolution apparatus due to the $\mathrm{pH}$ changes. Consequently, the in vitro dissolution results may not predict in vivo dissolution profile. The gastrointestinal simulator (GIS) is the in vitro dissolution apparatus to understand the in vivo dissolution phenomena of those drugs. The buffer solution with different $\mathrm{pH}$ in each compartment of the GIS will be transferred from one chamber to the next chamber with various transit rates. So it is important to understand the effect of different $\mathrm{pH}$ buffers $(1.2,1.5,4.5,6.8$ and 7.4$)$ on the solubility of the pioglitazone $\mathrm{HCl}$. With increase in $\mathrm{pH}$ of the buffer medium, the solubility of pioglitazone $\mathrm{HCl}$ decreased up to the $\mathrm{pH} 6.8$ may due to precipitation ${ }^{[33]}$. The solubility of pioglitazone is found to decrease drastically after $\mathrm{pH}$ 1.2. Further slight enhancement of solubility was observed at $\mathrm{pH} 7.4$, which may due to the supersaturation and recrystallization of drug ${ }^{[33,34]}$. Similar pattern was also seen in the three different samples. As seen from the fig. 2, SM showed highest solubility in all $\mathrm{pH}$ conditions among three different formulations. Higher amount of conversion of crystalline form of pioglitazone $\mathrm{HCl}$ into amorphous form might be the reason for the above. MM showed slightly better solubility of pioglitazone $\mathrm{HCl}$ than its corresponding PM. This may due to partial conversion into amorphous form during formulation in which some part of pioglitazone $\mathrm{HCl}$ was dissolved in the melted Solutol HS 15.

The dissolution profiles of pure drug, 1:7 PM and SDs prepared by $\mathrm{MM}$ and SM were evaluated in $0.1 \mathrm{~N} \mathrm{HCl}$ as depicted in the fig. 3. The dissolution of crystalline pioglitazone $\mathrm{HCl}$ was incomplete with only $43.7 \%$ after $60 \mathrm{~min}$. The PM showed $85 \%$ of drug dissolution within $45 \mathrm{~min}$ and incomplete drug release profile within $60 \mathrm{~min}$ of dissolution study. The enhancement of dissolution might be due to the solubilization of pioglitazone $\mathrm{HCl}$ by micellization.

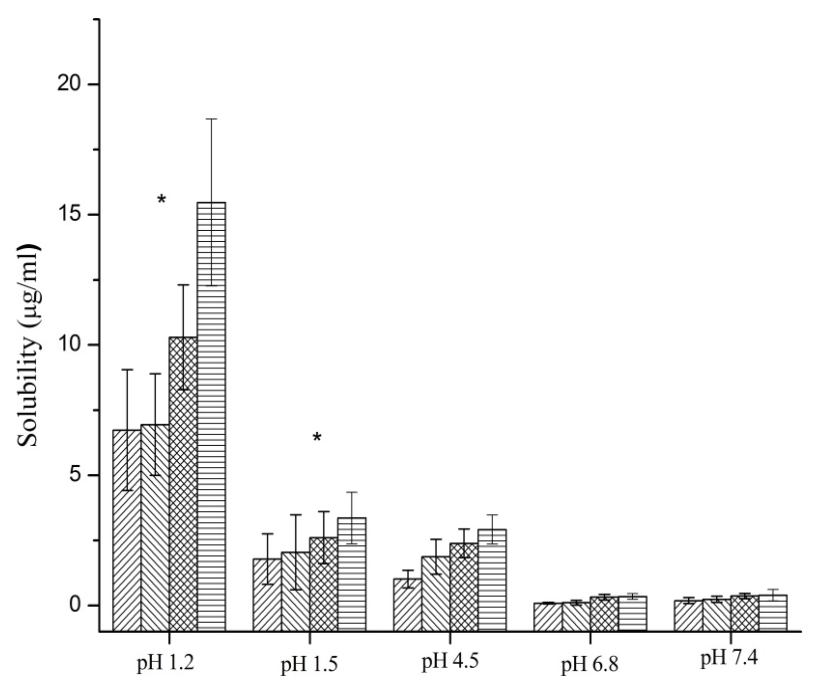

Fig. 2: Solubility of pioglitazone HCl from PMs and SDs in various pH buffers

Solubility of pioglitazone HCl from physical mixture (PM) and solid dispersions (SDs) prepared by melting method (MM) and solvent evaporation method (SM) in various $\mathrm{pH}$ buffers Mean \pm SD, $n=3,{ }^{*} \mathbf{p}<0.05$, TI/ pioglitazone, 1:7 PM, 1:7 MM, $\equiv 1: 7 \mathrm{SM}$

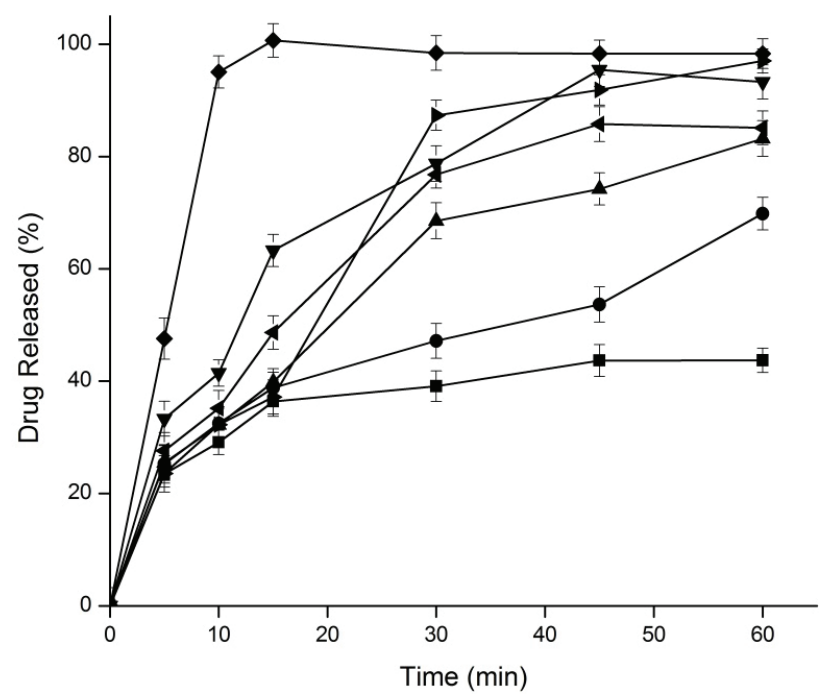

Fig. 3: Dissolution profiles of pioglitazone $\mathrm{HCl}, \mathrm{PM}$ and SD formulations in $0.1 \mathrm{~N} \mathrm{HCl}$

Dissolution profiles of pioglitazone $\mathrm{HCl}$, physical mixture (PM) and solid dispersion (SD) formulations prepared by melting method (MM) and solvent evaporation method (SM) using 1:3, 1:5 and 1:7 weight ratios in $0.1 \mathrm{~N} \mathrm{HCl}$. Mean \pm SD, $n=3$, (一匹-)

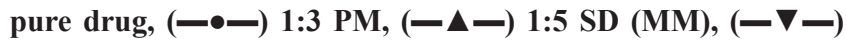
1:5 SD (SM), (一४-) 1:7 PM, (一-) 1:7 SD (MM), (一४) 1:7 SD (SM)

In 15 min of dissolution, complete drug release was observed for the formulation of SM but it decreased to $\sim 98 \%$ at $30 \mathrm{~min}$. The decrease in $\%$ of dissolution with increased in time interval could be due to precipitation of drug present in supersaturated state in buffer. Drug release $(37 \%)$ was observed for the formulation of MM within 15 min. Interestingly in PM, approximately 
$50 \%$ of pioglitazone $\mathrm{HCl}$ was released within a span of $15 \mathrm{~min}$ and it was slow and incomplete in the latter stage. Increased number of micellar concentration of the polymer might be the reason for sudden increase of pioglitazone $\mathrm{HCl}$.

At $15 \mathrm{~min}$ of dissolution, initial dissolution rate of Solutol HS 15-based SD (SM) and pure drug pioglitazone $\mathrm{HCl} 5.95$ and $2.43 \% / \mathrm{min}$, respectively. This was significantly $(p<0.05)$ higher than that of pure drug. The relative dissolution rate was found to be 2.44 . The dissolution efficiency of the sample improved from $53.97 \%$ (pure drug) to $60.13 \%$. This indicated enhancement in the dissolution rate of the drug with $\mathrm{SD}$. The $\mathrm{T}_{50 \%}$ of the pioglitazone $\mathrm{HCl}$ and formulation prepared in SM was found to be $>60$ and $7.27 \mathrm{~min}$. This indicates the significance difference $(p<0.05)$. Difference factor $\left(f_{1}\right)$ and similarity factor $\left(f_{2}\right)$ was calculated between the pioglitazone $\mathrm{HCl}$ and Solutol HS 15-based SD (SM) and found to be $123.93\left(f_{1}\right)$ and $16.17\left(\mathrm{f}_{2}\right)$. Pioglitazone $\mathrm{HCl}$ is not similar to Solutol HS 15 -based SD $(S M)$ product as the $f_{1}$ and $f_{2}$ values are more than 15 and less than 50, respectively. The effect of preparation methods of pioglitazone $\mathrm{HCl}$ in Solutol HS 15 increased the dissolution rate in the following order: $\mathrm{SM}>\mathrm{MM}>\mathrm{PM}>$ drug. Significant enhancement of dissolution rate observed in formulations of SM compared to PM and pure drug indicated pioglitazone $\mathrm{HCl}$ was present predominantly in an amorphous state in SM and this was confirmed by the data obtained in the DSC and XRD studies. Dissolution enhancement from SDs could be attributed to hydrophilicity, wettability, and formation of particles with higher porosity and properties of the polymers themselves ${ }^{[35]}$. Hence, Solutol HS 15 was suitable choice of polymer and SM was suitable method for dissolution enhancement of pioglitazone $\mathrm{HCl}$.

The FTIR spectra of pure drug pioglitazone $\mathrm{HCl}$, Solutol HS 15 and different formulations of pioglitazone $\mathrm{HCl}$ are depicted in fig. 4. The FTIR spectra of pioglitazone $\mathrm{HCl}$ showed characteristics absorption peak at $3354.67 \mathrm{~cm}^{-1}(\mathrm{~N}-\mathrm{H}$ stretching vibration), $3084.18 \mathrm{~cm}^{-1}$ (C-H aromatic vibration), $2927.94 \mathrm{~cm}^{-1}$ (C-H aliphatic stretching), 1741.72 $\mathrm{cm}^{-1}(\mathrm{C}=\mathrm{O}$ carbonyl stretching vibration) (capable to form hydrogen bond). $\mathrm{C}=\mathrm{C}$ stretching vibration at $1680 \mathrm{~cm}^{-1}, \mathrm{CH}_{2}$ deformation at $1462.04 \mathrm{~cm}^{-1}$, $\mathrm{C}-\mathrm{O}-\mathrm{Ar}$ group at $1244.09 \mathrm{~cm}^{-1}$ and $\mathrm{C}-\mathrm{H}$ bending at $1037.70 \mathrm{~cm}^{-1}$ are displayed by the pure drug ${ }^{[36,37]}$. The FTIR of Solutol HS 15 could favour the physical interaction with drug. PMs of drug and the Solutol HS 15 showed no new peaks or shifting of existing peaks, indicate no chemical interaction. This signifies the stability of the drug and polymers. SDs of pioglitazone $\mathrm{HCl}$ showed shifting of carbonyl stretching vibration of pioglitazone $\mathrm{HCl}$ to $1740.7 \mathrm{~cm}^{-1}(\mathrm{MM})$ and $1738.8 \mathrm{~cm}^{-1}$ (SM). This indicate the formation of hydrogen bonds between the pioglitazone $\mathrm{HCl}$ and Solutol HS 15 .

DSC thermogram of pure pioglitazone $\mathrm{HCl}$, Solutol HS 15, PM and SD formulations are shown in fig. 5. The free pioglitazone $\mathrm{HCl}$ exhibited a single sharp endothermic peak at $180.9^{\circ}$ corresponding to its melting point accompanied by an enthalpy of fusion $(\Delta H)$ of $33.7 \mathrm{~J} / \mathrm{g}$ confirming its crystallinity. The endotherm of Solutol HS 15 displayed melting peak at $32.2^{\circ[30]}$. The DSC thermogram of the PMs showed individual broad endothermic peaks corresponding to melting points of Solutol HS 15 and pioglitazone $\mathrm{HCl}$ indicating crystallinity of the drug was remained

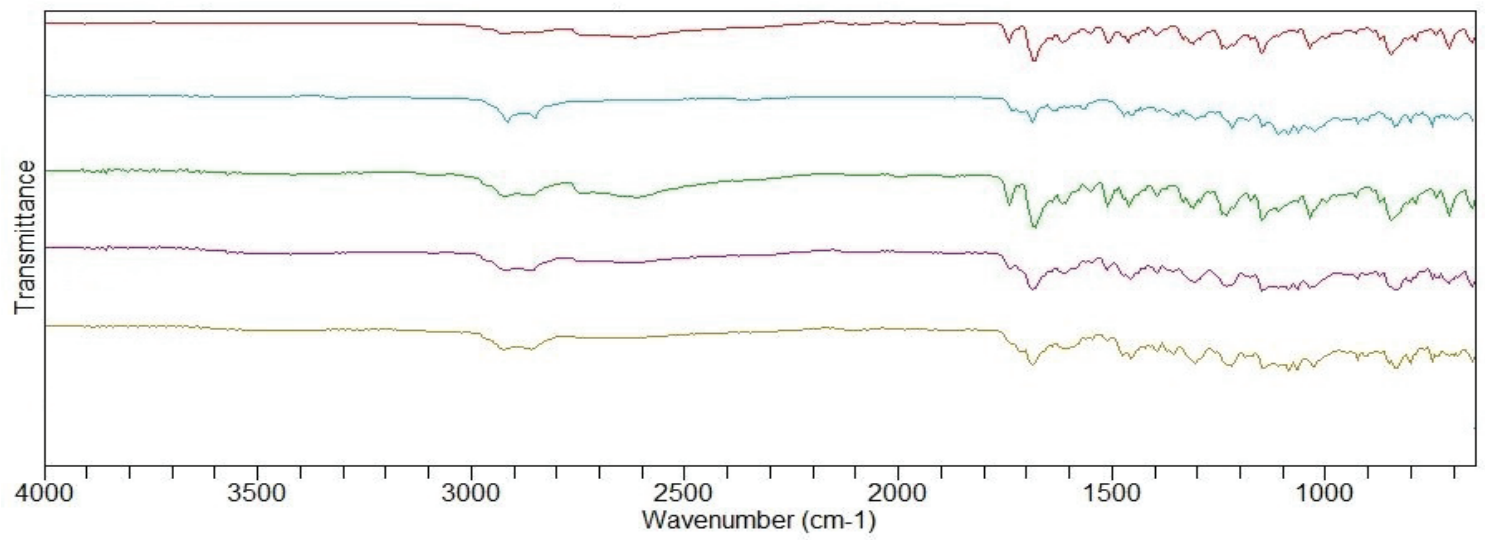

Fig. 4: FTIR spectra of pioglitazone HCl, Solutol HS 15, PM and SDs Physical mixture (PM) and solid dispersions (SDs) were prepared using 1:7 weight ratio melting method (MM) and solvent evaporation method (SM). (—) Pioglitazone HCI, (-) Solutol HS 15, (-) PM (1:7), ( -$)$ SD (1:7, MM) and (-) SD (1:7, SM) 
in the PMs. In the thermograms of SDs in MM, the endotherms of Solutol HS 15 were remain unchanged, while melting peak of pioglitazone $\mathrm{HCl}$ appeared with broad and less intensity at 1:5 and disappeared at 1:7 ratio indicating its partial and complete conversion into amorphous state, respectively (fig. 5). The absence of melting peak of pioglitazone $\mathrm{HCl}$ in the DSC thermogram of the prepared SDs (SM) at 1:5 and 1:7 ratio indicates a complete conversion of crystalline drug into amorphous form ${ }^{[23,29]}$. The results revealed that Solutol HS 15 have greater ability to form SDs in SM, which may result in higher dissolution rate and bioavailability.

The XRD pattern of pioglitazone $\mathrm{HCl}$, Solutol HS 15, $\mathrm{PM}, \mathrm{MM}$ and SM formulations were examined and depicted in fig. 6. Sharp and intense diffraction peak at $2 \theta^{\circ}$ equivalent to $6.87^{\circ}, 10.33^{\circ}, 13.8^{\circ}, 15.5^{\circ}$, $15.85^{\circ}, 16.99^{\circ}, 17.83^{\circ}, 18.95^{\circ}, 20.75^{\circ}, 21.72^{\circ}, 23.6^{\circ}$, $24.21^{\circ}, 25.42^{\circ}, 31.3^{\circ}$ showed the strong evidence of crystallinity of pioglitazone $\mathrm{HCl}$ in agreement to those of previous reports ${ }^{[15]}$. Solutol HS 15 exhibited no characteristics peaks as shown in fig. 6. The XRD peaks of pioglitazone $\mathrm{HCl}$ at $13.78^{\circ}, 15.85^{\circ}, 21.72^{\circ}$ disappeared in $\mathrm{PMs}$ and $18.95^{\circ}, 25.42^{\circ}$ peaks become shorter, implying that, drug was partially converted into amorphous form. The $2 \theta$ XRD peaks at $6.87^{\circ}$, $16.99^{\circ}, 17.83^{\circ}, 24.21^{\circ}, 25.42^{\circ}$ of pioglitazone $\mathrm{HCl}$ was appeared in the 1:5 SDs indicating drug still retained its crystallinity. The amount of Solutol HS 15 was not sufficient to solubilise the drug completely in both the techniques. The melting mixture at 1:7 ratio showed similar peaks as 1:5 SDs. On other hand, SDs (SM)

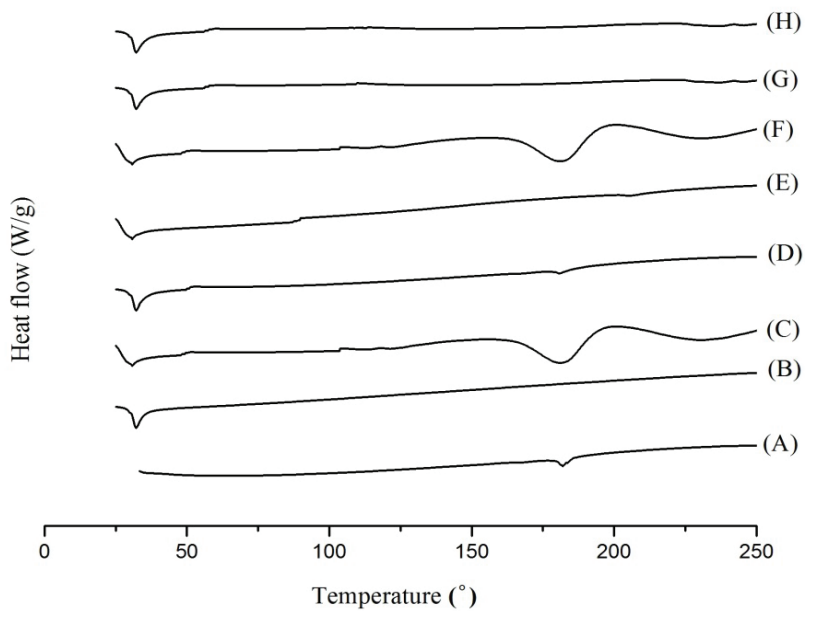

Fig. 5: DSC thermograms

A. pioglitazone HCl, B. Solutol HS 15, C. Solutol HS 15-based PM (1:3), D. Solutol HS 15-based SD (1:5, MM), E. Solutol HS 15-based SD (1:5, SM), F. Solutol HS 15-based PM (1:7), G. Solutol HS 15-based SD (1:7, MM), H. Solutol HS 15-based SD $(1: 7$, SM)

March-April 2019

Indian Journal of Pharmaceutical Sciences

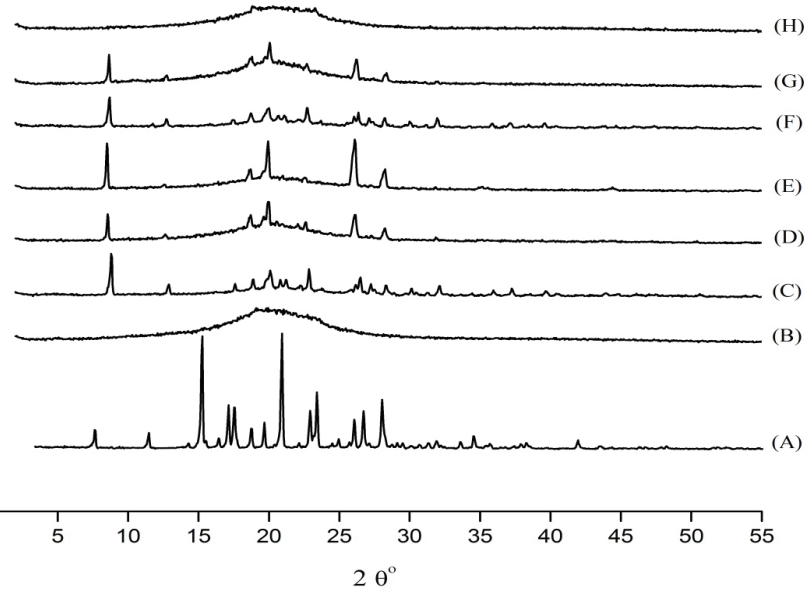

Fig. 6: X-ray diffraction patterns

A. Pioglitazone HCl, B. Solutol HS 15, C. Solutol HS 15-based PM (1:3), D. Solutol HS 15-based SD (1:5, MM), E. Solutol HS 15-based SD (1:5, SM), F. Solutol HS 15-based PM (1:7), G. Solutol HS 15-based SD (1:7, MM), H. Solutol HS 15-based SD $(1: 7, \mathrm{SM})$

at $1: 7$ ratio, didn't show any characteristics peaks of pioglitazone $\mathrm{HCl}$, suggesting complete change of drug crystallinity to amorphous state.

The higher solubility of SDs is primarily attributed to its conversion to amorphous state. This state being thermodynamically unstable, might convert to lower energy state by recrystallization during storage. Hence, rate of recrystallization and its effect of solubility of 1:7 SD prepared by SM was investigated by periodic evaluation under $25^{\circ}$ storage conditions according to the ICH guidelines. As shown in fig. 7 (I), 1:7 SD showed $385 \mu \mathrm{g} / \mathrm{ml}$ solubility of pioglitazone $\mathrm{HCl}$ in 0 day and it was maintained at this level during 3 mo of storage period. This indicates no significance difference $(p>0.05)$. This indicated that amorphous state of pioglitazone $\mathrm{HCl}$ in $\mathrm{SD}$ almost remained unchanged within the study period. After 3 mo of storage, XRD pattern fig. 7 (II), of SD formulation did not show any additional peaks, indicated drug remaining in the amorphous state. This confirmed that there is no recrystallization, which suggests its good physical stability.

To assess whether the in vitro dissolution parameters could be used to predict in vivo performance, a pharmacokinetic study was performed for the selected formulation and pure drug in rabbits. The plasma concentration-time curves after oral administration of pure drug and formulation were shown in fig. 8 and the essential pharmacokinetics parameters were summarized in the Table 2. No significant difference was observed for $\mathrm{T}_{\max }$ and $\mathrm{T}_{1 / 2}$ between pure drug and 


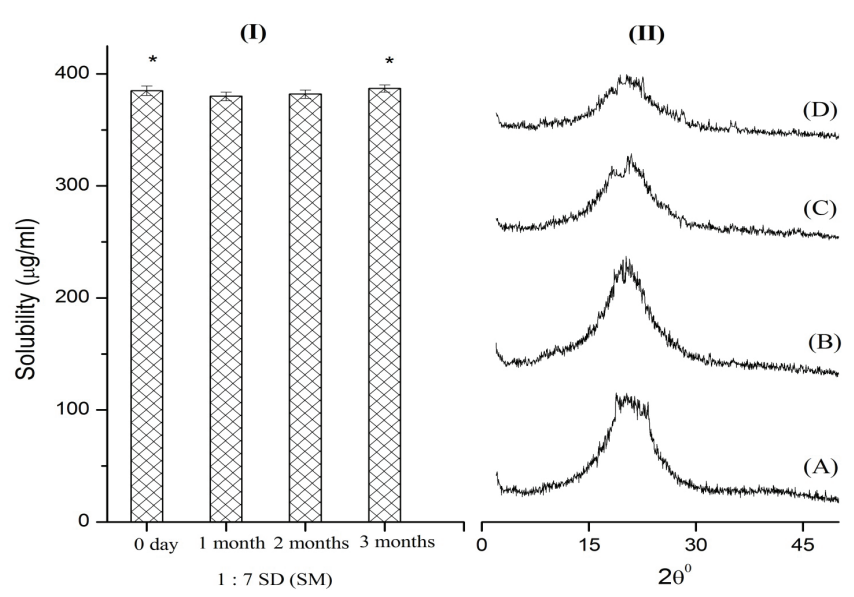

Fig. 7: Effect of storage at $25^{\circ}$ for 3 months on solubility and X-ray diffraction patterns of pioglitazone $\mathrm{HCl}$ from SDs

(I) Solubility of pioglitazone $\mathrm{HCl}$ from SDs prepared by solvent evaporation method (SM) during 3 months storage at $25^{\circ}$, Mean $\pm S D, n=3$, "p<0.05; (II) $X$-ray diffraction patterns, $A$. Solutol HS 15-based SD (1:7) at 0 day, B. Solutol HS 15-based SD (1:7) after 1 month, C. Solutol HS 15-based SD (1:7) after 2 months, D. Solutol HS 15-based SD (1:7) after 3 months at $25^{\circ}$

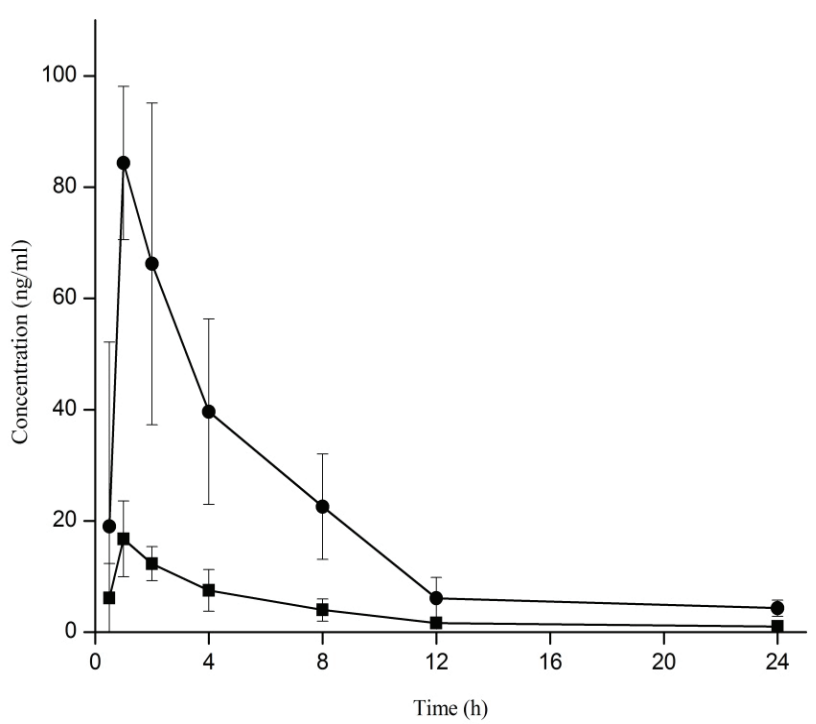

Fig. 8: Plasma concentration of pioglitazone $\mathrm{HCl}$ with time from pioglitazone $\mathrm{HCl}$ and SD of pioglitazone

Plasma concentration profile of pioglitazone $\mathrm{HCl}$ and SD of pioglitazone $\mathrm{HCl}$ with Solutol HS 15 prepared by solvent evaporation method (SM) Mean \pm SD, $n=6$, (- (一॰-) 1:7 SD (SM)

TABLE 2: PHARMACOKINETIC PARAMETERS OF PIOGLITAZONE HCI AND SOLUTOL HS 15-BASED SD (1:7) IN RABBITS

\begin{tabular}{lcc}
\hline Parameter & Pioglitazone HCl & $\begin{array}{c}\text { Solutol HS 15-based } \\
\text { SD }\end{array}$ \\
\hline $\mathrm{T}_{\max }(\mathrm{h})$ & $1.00 \pm 0.001$ & $0.83 \pm 0.02$ \\
$\mathrm{C}_{\max }(\mathrm{ng} / \mathrm{ml})$ & $16.78 \pm 6.81$ & $64.37 \pm 5.18^{*}$ \\
$\mathrm{AUC}_{0-\mathrm{t}}(\mathrm{ng} \mathrm{h} / \mathrm{ml})$ & $91.52 \pm 37.18$ & $216.37 \pm 52.61^{*}$ \\
$\mathrm{AUC}_{0-\infty}(\mathrm{ng} \mathrm{h} / \mathrm{ml})$ & $99.54 \pm 38.19$ & $221.51 \pm 53.60^{*}$ \\
$M R T(\mathrm{~h})$ & $8.52 \pm 0.87$ & $4.71 \pm 0.45^{*}$ \\
$\mathrm{~T}_{1 / 2}(\mathrm{~h})$ & $5.73 \pm 0.40$ & $4.07 \pm 0.29$ \\
\hline
\end{tabular}

Mean $\pm S D,(n=3),{ }^{*} p<0.05$ compared to pioglitazone $\mathrm{HCl}$
Solutol HS 15-based 1:7 SD after oral administration. In contrast to this, $\mathrm{C}_{\max }$ of $\mathrm{SD}$ formulation was significantly higher (approximately 4 fold) than that of crystalline pioglitazone $\mathrm{HCl}(\mathrm{p}<0.05)$. This revealed the increase in bioavailability of pioglitazone $\mathrm{HCl}$ from SD preparation. In agreement with this, $\mathrm{AUC}_{0-\mathrm{t}}$ of the SD was found to be about 2.4 fold higher than that of pure drug $(p<0.05)$. These findings suggest the improvement in oral bioavailability of pioglitazone $\mathrm{HCl}$ after administration of SD. This may due to excellent solubilization capacity of Solutol HS 15 and conversion to amorphous state during SD preparation by SM. Mean residence time of formulations showed more than the pure drug.

This study revealed that Solutol HS 15 is an effective carrier in enhancing solubility and stability of the pioglitazone $\mathrm{HCl}$. Pioglitazone $\mathrm{HCl}$ in the SD prepared by SM with Solutol HS 15 at weight ratio of 1:7 existed in amorphous state and was stable under tested storage condition. This has resulted in significant improvement in the bioavailability. Thus, the present study showed an effective way of overcoming problems of solubility, stability and oral bioavailability of pioglitazone $\mathrm{HCl}$. Considering the simplicity of this approach, it could further be utilized to design capsules in higher scale for industrial application.

\section{Acknowledgements:}

The authors would like to thank Aurobindo Pharma Ltd., Hyderabad, India for providing the pure drug pioglitazone $\mathrm{HCl}$ as gift sample.

\section{Conflict of interest:}

The authors declare no conflict of interest.

\section{REFERENCES}

1. Pretorius E, Bouic PJ. Permeation of four oral drugs through human intestinal mucosa. AAPS PharmSciTech 2009;10(1):270-5.

2. Gomez-Orellana I. Strategies to improve oral drug bioavailability. Expert Opin Drug Deliv 2005;2(3):419-33.

3. Olefsky JM. Treatment of insulin resistance with peroxisome proliferator-activated receptor gamma agonists. J Clin Invest 2000;106(4):467-72.

4. Gillies PS, Dunn CJ. Pioglitazone. Drugs 2000;60(2):333-43.

5. Waugh J, Keating GM, Plosker GL, Easthope S, Robinson DM. Pioglitazone: A review of its use in type 2 diabetes mellitus. Drugs 2006;66(1):85-109.

6. Taupitz T, Dressman JB, Klein S. New formulation approaches to improve solubility and drug release from 
fixed dose combinations: Case examples pioglitazone/ glimepiride and ezetimibe/simvastatin. Eur J Pharm Biopharm 2013;84(1):208-18.

7. Elbary AA, Kassem MA, Abou SM, Khalil RM. Formulation and hypoglycemic activity of pioglitazone-cyclodextrin inclusion complexes. Drug Discov Ther 2008;2(2):94-107.

8. Pandit V, Gorantla R, Devi K, Pai RS, Sarasija S. Preparation and characterization of pioglitazone cyclodextrin inclusion complexes. J Young Pharm 2011;3(4):267-74.

9. Beloshe S, Chougule D, Shah R, Ghodke D, Pawar N, Ghaste R. Effect of method of preparation on pioglitazone HCl- $\beta$-cyclodextrin inclusion complexes. Asian J Pharm 2010;4(2):168-72.

10. Mummidi V, Jayanthi V. Effect of hydrophilic polymers on isradipine complexation with hydroxypropyl $\beta$-cyclodextrin. Drug Dev Ind Pharm 2013;39(7):970-77.

11. Shafiq-un-Nabi S, Shakeel F, Talegaonkar S, Ali J, Baboota $\mathrm{S}$, Ahuja A, et al. Formulation development and optimization using nanoemulsion technique: a technical note. AAPS PharmSciTech 2007;8(2):E12-E17.

12. Seedher N, Kanojia M. Co-solvent solubilization of some poorly-soluble antidiabetic drugs. Pharm Dev Technol 2009;14(2):185-92.

13. Jahnavi N, Dixit A, Mehta AK, Mohan VM. Formulation, evaluation and characterization of solid dispersions of pioglitazone hydrochloride. Indian $\mathrm{J}$ Pharm Educ Res 2013;47(2):113-22.

14. Jouyban A, Soltanpour S. Solubility of pioglitazone hydrochloride in binary mixtures of polyethylene glycol 400 with ethanol, propylene glycol, N-methyl-2-pyrrolidone, and water at 25 degrees C. Chem Pharm Bull 2010;58(9):1132-5.

15. Shi NQ, Lei YS, Song LM, Yao J, Zhang XB, Wang XL. Impact of amorphous and semicrystalline polymers on the dissolution and crystallization inhibition of pioglitazone solid dispersions. Powder Technol 2013;247:211-21.

16. Pokharkar V, Kutwal M, Mandpe L. Pioglitazone solid dispersion system prepared by spray drying method: in vitro and in vivo evaluation. PDA J Pharm Sci Technol 2013;67(1):23-34.

17. Mooter GV den. The use of amorphous solid dispersions: A formulation strategy to overcome poor solubility and dissolution rate. Drug Discov Today Technol 2012;9(2):e79-e85.

18. Shamma RN, Basha M. Soluplus: A novel polymeric solubilizer for optimization of Carvedilol solid dispersions: Formulation design and effect of method of preparation. Powder Technol 2013;237:406-14.

19. Song CK, Yoon IS, Kim DD. Poloxamer-based solid dispersions for oral delivery of docetaxel: Differential effects of F68 and P85 on oral docetaxel bioavailability. Int J Pharm 2016;507(1-2):102-8.

20. Corrigan OI, Healy AM. Surfactants in pharmaceutical products and systems. In: Swarbrick J, Boylan JC, editors. Encyclopedia of Pharmaceutical Technology. 3rd ed. New York: Informa Healthcare; 2006. p. 3583-96.

21. Vo CLN, Park C, Lee BJ. Current trends and future perspectives of solid dispersions containing poorly water-soluble drugs. Eur J Pharm Biopharm 2013;85(3 part B):799-813.

22. Leuner C, Dressman J. Improving drug solubility for oral delivery using solid dispersions. Eur J Pharm Biopharm 2000;50(1):47-60.

23. Ansari MT, Arshad MS, Hussain A, Ahmad Z. Improvement of solubility, dissolution and stability profile of artemether solid dispersions and self-emulsified solid dispersions by solvent evaporation method. Pharm Dev Technol 2016;23(10):1-9.

24. ICH, guidance for industry: stability testing of new drug substances and products ICH Q1A (R2), 2003. [cited 2016 Jan 10]. Available from: http://www.fda.gov/cber/gdlns/ichsta. pdf.

25. Alam S, Aslam M, Khan A, Imam SS, Aqil M, Sultana Y, et al. Nanostructured lipid carriers of pioglitazone for transdermal application: from experimental design to bioactivity detail. Drug Deliv 2016;23(2):601-9.

26. Nair AB, Jacob S. A simple practice guide for dose conversion between animals and human. J Basic Clin Pharm 2016;7(2):2731 .

27. Zhang Y, Huo M, Zhou J, Xie S. PKSolver: An add-in program for pharmacokinetic and pharmacodynamic data analysis in Microsoft Excel. Comput Methods Programs Biomed 2010;99(3):306-14.

28. Bolton S, Bon C. Pharmaceutical statistics: practical and clinical applications. New York: Informa Healthcare; 2010.

29. Han HK, Lee BJ, Lee HK. Enhanced dissolution and bioavailability of biochanin A via the preparation of solid dispersion: In vitro and in vivo evaluation. Int $\mathrm{J}$ Pharm 2011;415(1-2):89-94.

30. Seo SW, Han HK, Chun MK, Choi HK. Preparation and pharmacokinetic evaluation of curcumin solid dispersion using solutol ${ }^{\circledR}$ HS15 as a carrier. Int J Pharm 2012;424(12):18-25.

31. Tantishaiyakul V, Kaewnopparat N, Ingkatawornwong S. Properties of solid dispersions of piroxicam in polyvinylpyrrolidones. Int J pharm 1999;181(2):143-51.

32. Bravo González RC, Boess F, Durr E, Schaub N, Bittner B. In vitro investigation on the impact of Solutol HS 15 on the uptake of colchicine into rat hepatocytes. Int J Pharm 2004;279(12):27-31.

33. Eckland DA, Danhof M. Clinical pharmacokinetics of pioglitazone. Exp Clin Endocrinol Diabetes 2000;108:S234-S242.

34. Patel BB, Patel JK, Chakraborty S. Solubility enhancement using poly(meth)acrylate based solid dispersions. Powder Technol 2015;270(part A):27-38.

35. Baghel S, Cathcart H, O'Reilly NJ. Polymeric amorphous solid dispersions: a review of amorphization, crystallization, stabilization, solid-state characterization, and aqueous solubilization of biopharmaceutical classification system class II drugs. J Pharm Sci 2016;105(9):2527-44.

36. Chowdary YA, Raparla R, Madhuri M. Formulation and evaluation of multilayered tablets of pioglitazone hydrochloride and metformin hydrochloride. J Pharm 2014;2014:848243.

37. Mehatha AK, Suryadevara V, Lankapalli SR, Deshmukh AM, Sambath LP. Formulation and optimization of pioglitazone solid dispersions prepared by hot melt extrusion technique. Indonesian J Pharm 2013;24(3):206-14. 